\title{
Efeito de elevadas produtividades do vinhedo nas características físico-químicas e sensoriais do vinho Merlot
}

\author{
Effect of high vineyard yields on the physicochemical and sensory characteristics of the Merlot wine
}

\author{
Alberto Miele $^{1}$ Luiz Antenor Rizzon ${ }^{2}$
}

\section{RESUMO}

\begin{abstract}
$O$ objetivo do trabalho foi determinar o efeito de elevadas produtividades dos vinhedos nas características físicoquímicas e sensoriais do vinho Merlot jovem produzido em região de montanha. $O$ experimento foi realizado durante o ciclo vegetativo da videira de 1999/2000, constando de quatro tratamentos (produtividades do vinhedo - $\mathrm{kg} \mathrm{ha}^{-1}-22.400$, $27.800,34.700$ e 44.900) e três repetições; cada parcela foi constituída de cinco plantas. A uva foi colhida em fevereiro de 2000, por ocasião de sua maturação, e levada ao Laboratório de Microvinificação onde foi vinificada em recipientes de vidro de 20L. Avaliaram-se as variáveis físico-químicas por processos clássicos e as sensoriais por um grupo de degustadores utilizando-se uma ficha de degustação não-paramétrica. Os dados obtidos foram submetidos à análise de regressão polinomial. Os resultados mostram que a produtividade crescente do vinhedo não teve efeito significativo sobre as variáveis físico-químicas. Entretanto ela foi significativa sobre as variáveis visuais, olfativas e gustativas. Os vinhos elaborados com uvas provenientes de vinhedos com a menor produtividade apresentaram-se visualmente com matiz vermelho-violáceo mais acentuado; mais francos, finos e típicos no olfato; e com gosto mais equilibrado, típicos, frutados e mais encorpados. Os vinhos de média produtividade tiveram cor mais intensa; olfato também mais intenso, mas com notas vegetais e de pimentão verde mais acentuadas; gosto mais intenso. As demais variáveis sensoriais não foram afetadas pela produtividade do vinhedo.
\end{abstract}

Palavras-chave: uva, análise sensorial, degustação, viticultura, enologia.

\section{ABSTRACT}

The objective of the work was to determine the effect of high vineyard yields on the physicochemical and sensory characteristics of the young Merlot wine produced in a mountainous region. The experiment was conducted during the 1999/2000 grapevine vegetative cycle. It had four treatments (vineyard yields - $k g h a^{-1}-22,400,27,800,34,700$ and 44,900) and three replications; each parcel was constituted by five grapevines. Ripe grapes were harvested in February of 2000 and transported to the Microvinification Laboratory where they were processed in $20 \mathrm{~L}$ glass recipients. Physicochemical variables were determined by the classic methods and sensory analysis was performed by a panel using a non-parametric tasting sheet. Data were submitted to the polynomial regression analysis. The results show that increasing yields did not have any effect on the physicochemical variables. However, it showed significant effect on the variables related to vision, smell and taste. Wines made with grapes from vineyards having the smaller yield showed a more pronounced red-violet color; in the smell they were cleaner, elegant and had higher tipicity; concerning to the taste, they showed a higher equilibrium, tipicity, fruit notes and body. Those from average yields had a more intense color; smell more intense too, but with vegetal and green pepper characters more pronounced; they were more intense in taste. The other variables were not affected by the vineyard yield.

Key words: grape, tasting, sensory analysis, viticulture, enology.

\section{INTRODUÇÃO}

Em geral, constata-se uma relação significativa e negativa entre a produtividade do vinhedo e a qualidade da uva e, conseqüentemente, do vinho (WINKLER, 1954). Tem-se verificado, entretanto, que esta relação, em muitos casos, não é linear, mas quadrática do tipo $\mathrm{Y}=\mathrm{a}+\mathrm{bX}-\mathrm{cX}^{2}$, ou seja, os melhores vinhos são elaborados com uvas provenientes de vinhedos com produtividades intermediárias, excluindo-se os extremos de baixa e alta produtividades (WINKLER, 1954; COLLALTO et al.,

\footnotetext{
${ }^{1}$ Empresa brasileira de Pesquisa Agropecuária (Embrapa) Uva e Vinho, 95700-000, Bento Gonçalves, RS, Brasil. E-mail: miele@cnpuv.embrapa.br. Autor para correspondência.

${ }^{2}$ Embrapa Uva e Vinho, 95700-000 Bento Gonçalves, RS, Brasil. E-mail: rizzon@cnpuv.embrapa.br.
} 
1991; KIEFER \& WEBER, 1994; SCHALKWYK et al., 1995; BOULTON et al., 1998; GRASSL et al., 2002; NAOR et al., 2002). Este comportamento ocorre especialmente em regiões vitícolas caracterizadas por clima quente e por solos que apresentam composição química e reserva hídrica pobre. Mas esta relação não é evidente em todas as regiões vitícolas do mundo, pois há resultados que mostram que o aumento do teor de açúcar no mosto da uva não é diretamente proporcional à diminuição da produtividade do vinhedo (FREEMAN, 1983; OUGH \& NAGAOKA, 1984; BRAVDO et al., 1985). Há, inclusive, trabalhos que concluem que o aumento da produtividade do vinhedo tem um efeito pequeno, ou mesmo nenhum, sobre a diminuição da qualidade do vinho (LOINGER \& SAFRAN, 1971; BRAVDO et al., 1984; EAWART et al., 1985; REYNOLDS et al., 1986, LAVIN et al., 2001).

O clima da Serra Gaúcha, a mais importante região vitivinícola do Brasil, em geral se caracteriza por precipitações pluviométricas relativamente elevadas, o que torna a terra úmida, e por solos com elevados teores de matéria orgânica. Essas condições diminuem a possibilidade da uva Merlot exteriorizar todo seu potencial genético para atingir uma maturação ideal para elaborar vinhos de qualidade e com características específicas determinadas pelo produtor. Tem-se que considerar, ainda, que cada viticultor cultiva, em média, 2,0ha de vinhedos. Esta superfície é pequena para que ele tenha uma rentabilidade econômica compatível com seu trabalho familiar e suas necessidades. Essas condições, por si só, justificam práticas vitícolas que favoreçam produtividades dos vinhedos relativamente elevadas.

Face a esta realidade, conduziu-se este estudo com o objetivo de determinar o efeito de produtividades elevadas do vinhedo sobre as características físico-químicas e sensoriais do vinho jovem Merlot em função das condições edafoclimáticas da Serra Gaúcha.

\section{MATERIAL E MÉTODOS}

O trabalho foi realizado durante o ciclo vegetativo da videira de 1999/2000, em um vinhedo da cv. Merlot. Este vinhedo foi formado em 1983, a partir de plantas enxertadas sobre o porta-enxerto Paulsen 1.103, provenientes de um viveirista da França. O vinhedo foi conduzido no sistema de latada e o espaçamento entre fileiras foi de 3,0m e entre plantas, de 2,0m. As videiras foram podadas no sistema Guyot.
O protocolo experimental teve quatro tratamentos, constituídos por quatro níveis médios de produtividade $\left(\mathrm{kg} \mathrm{ha}^{-1}\right)$ - 22.400, 27.800, 34.700 e $44.900-$, determinados pela carga de gemas/ha. O rendimento de $22.400 \mathrm{~kg} \mathrm{ha}^{-1}$ foi considerado como testemunha, pois foi similar à média anual deste vinhedo. Houve três repetições, sendo cada parcela formada por cinco plantas. O delineamento experimental foi em blocos ao acaso.

Com a maturação fisiológica da videira, as uvas foram colhidas, transportadas ao Laboratório e imediatamente microvinificadas. A elaboração do vinho de cada parcela foi feita com $18 \mathrm{~kg}$ de uva as quais, após terem sido desengaçadas e esmagadas, foram colocadas em recipientes de vidro com capacidade de 20L. A seguir, adicionaram-se $50 \mathrm{mg} \mathrm{L}^{-1}$ de $\mathrm{SO}_{2}$ e leveduras secas ativas (Saccharomyces cerevisiae) na proporção de $0,2 \mathrm{~g} \mathrm{~L}^{-1}$ de mosto. Esses recipientes foram fechados com válvulas de Müller, de maneira que a fermentação do mosto se processasse em condições anaeróbias. Foram, então, colocadas em sala com temperatura controlada de $25^{\circ} \mathrm{C}$. A maceração da uva foi de seis dias, com uma remontagem/dia. Os mostos não foram chaptalizados. A descuba foi feita com uma leve prensagem do bagaço, o que permitiu a elaboração do vinho principalmente com a fase líquida. Concluídas as fermentações alcoólica - constatada pela análise da concentração de açúcar residual e pelo desprendimento de dióxido de carbono - e da malolática - constatada através de cromatografia de papel -, fez-se uma trasfega a fim de separar o vinho da borra. A estabilização do vinho foi realizada a uma temperatura de $-3^{\circ} \mathrm{C}$, durante dez dias. Estabilizado, o vinho foi engarrafado e conservado à $18^{\circ} \mathrm{C}$.

As análises físico-químicas dos vinhos densidade, álcool, acidez total, acidez volátil, acidez fixa, $\mathrm{pH}$, extrato seco, açúcares redutores, extrato seco reduzido, relação álcool/extrato seco reduzido, cinzas, alcalinidade das cinzas e dióxido de enxofre total e livre-, foram determinadas pela metodologia de RIBÉREAUGAYON et al. (1976); as antocianinas, pelo método de diferença de pH (RIBÉREAU-GAYON \& STONESTREET, 1965). As demais análises foram feitas através da metodologia de RIBÉREAU-GAYON \& STONESTREET (1966): os taninos, por hidrólise ácida; a densidade ótica, pelo comprimento de onda a 420 , 520 e $620 \mathrm{~nm}$, a intensidade de cor e o matiz utilizando um espectrofotômetro UV/VIS e cubetas de $1 \mathrm{~mm}$ de percurso ótico $\mathrm{pH}$.

A análise sensorial foi realizada por um grupo de degustadores experimentados, utilizando uma ficha de degustação com escala não estruturada (Figura 1), com extremos de $100 \mathrm{~mm}$ de comprimento. As 
Nome:

Data:

Amostra:

Favor colocar um traço vertical no espaço correspondente a cada variável indicada. Não deixar nenhuma variável em branco.

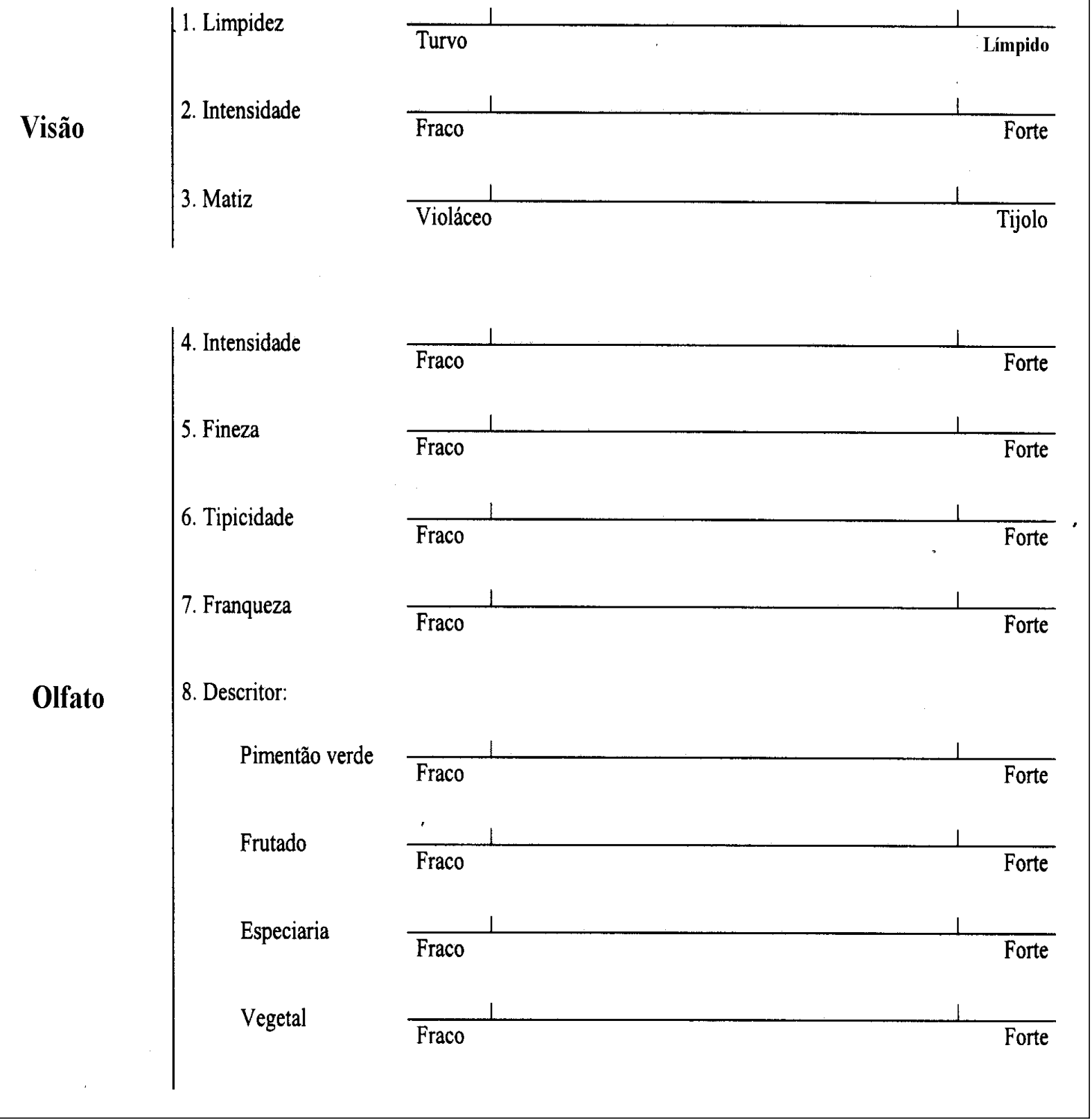


Figura 1 - continuação

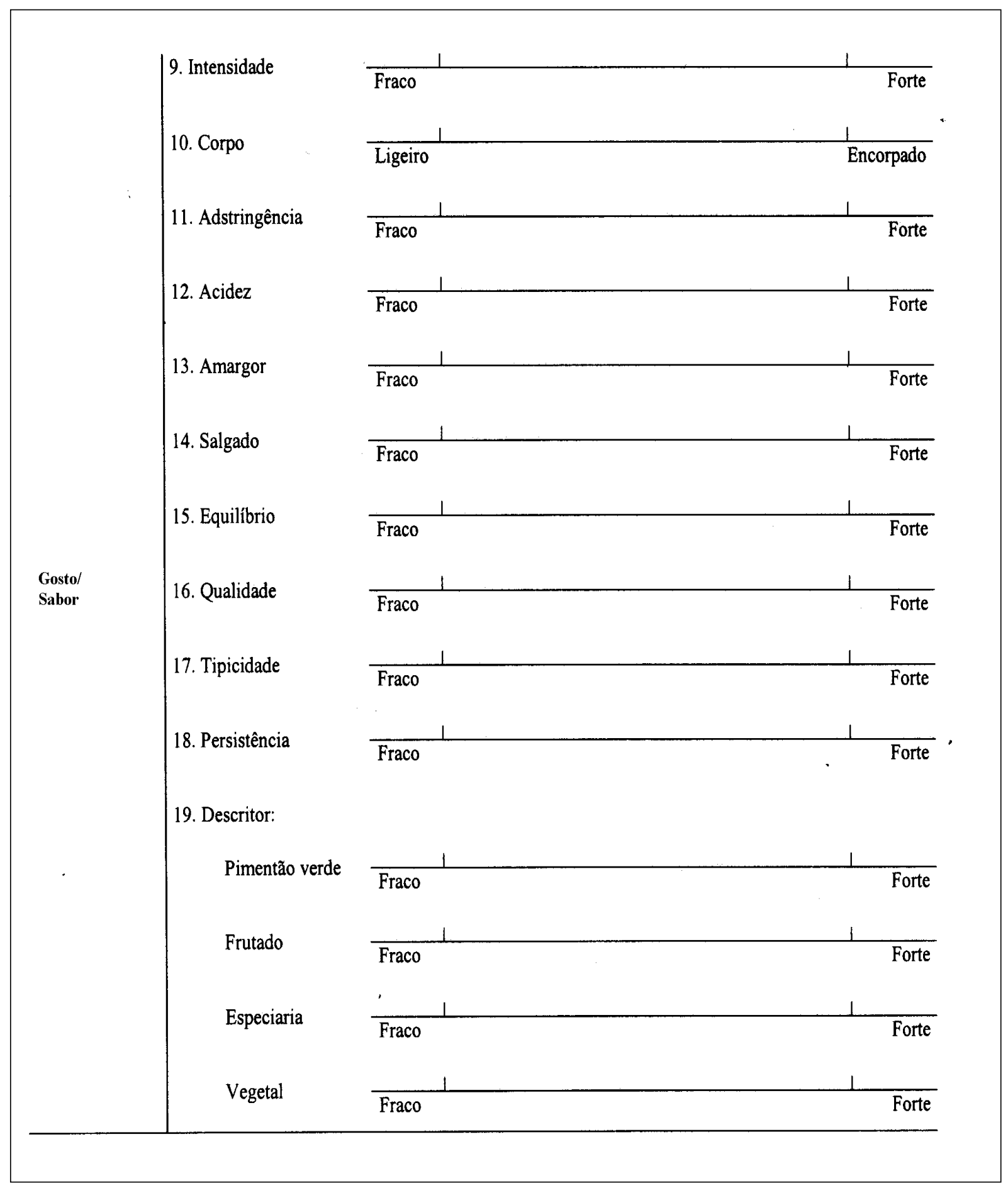

Figura 1 - Ficha de análise sensorial não-paramétrica para vinho tinto.

Ciência Rural, v.36, n.1, jan-fev, 2006. 
variáveis avaliadas foram: 1) Visão $\Rightarrow \operatorname{limpidez~(VLI),~}$ intensidade (VIN), matiz (VMA); 2) Olfato $\Rightarrow$ intensidade (OIN), fineza (OFI), tipicidade (OTI), franqueza (OFA), pimentão verde (OPV), frutado (OFR), especiaria (OEP), vegetal (OVE); 3) Gosto/Sabor $\Rightarrow$ intensidade (GIN), corpo (GCP), adstringência (GAS), acidez (GAC), amargor (GAM), salgado (GSA), equilíbrio (GEQ), qualidade (GQU), tipicidade (GTI), persistência (GPE), pimentão verde (GPV), frutado (GFR), especiaria (GEP) e vegetal (GVE).

Os resultados das análises físico-químicas e sensoriais do vinho Merlot foram submetidos à análise de regressão polinomial a 5\% de probabilidade de erro.

\section{RESULTADOS E DISCUSSÃO}

Os resultados mostram que a produtividade crescente do vinhedo não teve efeito significativo sobre as variáveis físico-químicas do vinho Merlot jovem (Tabela 1). Esses resultados, sem dúvida, impressionam, pois houve uma diferença de $22.500 \mathrm{~kg} \mathrm{ha}^{-1}$ de uva entre a maior e a menor produtividade e esta diferença não influenciou na concentração de açúcar do mosto e, conseqüentemente, no teor de álcool do vinho.

Entretanto, a análise de regressão polinomial evidenciou efeitos significativos numa parte das variáveis sensoriais (Tabela 2). Esse efeito foi linear para algumas variáveis e quadrático para outras. Os vinhos elaborados com uvas provenientes de vinhedos com a menor produtividade apresentaram-se visualmente com matiz vermelho-violáceo (VMA) mais acentuado; no olfato, foram francos (OFA), mais finos (OFI) e mais típicos (OTI); no gosto, mais encorpados (GCP), equilibrados (GEQ), típicos (GTI) e frutados
(GFR). Os vinhos de média produtividade tiveram cor mais intensa (VIN); olfato também mais intenso (OIN), mas com notas vegetais (OVE) e de pimentão verde (OPV) mais acentuadas; e gosto mais intenso (GIN). As demais variáveis sensoriais não foram afetadas pela produtividade do vinhedo.

Esses resultados mostram que a análise sensorial se constitui em poderoso auxiliar na avaliação dos vinhos, pois detectou diferenças significativas entre os tratamentos enquanto que o mesmo não ocorreu em relação às análises físico-químicas.

Em geral, visualmente os vinhos Merlot jovens (Figura 2) apresentaram-se límpidos (VLI), possuíam uma intensidade de cor (VIN) relativamente forte e matiz (VMA) vermelho-violáceo. Com relação ao aspecto olfativo, eram relativamente francos (OFA), intensos (OIN) e com certa fineza (OFI) e certa tipicidade (OTI); os descritores frutado (OFR) e especiaria (OEP) eram moderados, enquanto que as notas de vegetal (OVE) e de pimentão verde (OPV) foram pouco pronunciadas. No gosto/sabor, apresentaram-se com intensidade (GIN) e corpo (GCP), equilíbrio (GEQ), tipicidade (GTI), persistência (GPE) e qualidade (GQU) médias a forte; eram pouco adstringentes (GAS), os gostos amargo (GAM) e salgado (GSA) foram muito pouco percebidos; o caráter frutado (GFR), especialmente de frutas vermelhas não muito maduras, e os de especiaria (GEP), de vegetal (GVE) e de pimentão verde (GPE) foram fracos.

$\mathrm{O}$ efeito da produtividade do vinhedo nas características sensoriais do vinho Merlot jovem pode ter sido influenciado por vários fatores que regulam a fisiologia da videira e conseqüentemente a qualidade da uva e do vinho. Dentre esses fatores, citam-se as características do solo, o clima e o microclima, a radiação

Tabela 1 - Dados médios das variáveis físico-químicas dos quatro tratamentos do vinho Merlot jovem em função de crescentes produtividades do vinhedo. Bento Gonçalves, RS, $2000^{1}$

\begin{tabular}{llll}
\hline Variável & Média & Variável & Média \\
\hline Densidade & $0,9942 \mathrm{~g} \mathrm{~mL}^{-1}$ & Alcalinidade das cinzas & $22,8 \mathrm{meq} \mathrm{L}^{-1}$ \\
Álcool & $11,32 \% \mathrm{v} / \mathrm{v}$ & Dióxido de enxofre total & $21,9 \mathrm{mg} \mathrm{L}^{-1}$ \\
Acidez total & $75,1 \mathrm{meq} \mathrm{L}^{-1}$ & Dióxido de enxofre livre & $16,8 \mathrm{mg} \mathrm{L}^{-1}$ \\
Acidez volátil & $8,9 \mathrm{meq} \mathrm{L}^{-1}$ & Antocianinas & $79,0 \mathrm{mg} \mathrm{L}^{-1}$ \\
Acidez fixa & $65,6 \mathrm{meq} \mathrm{L}^{-1}$ & Taninos & $1,58 \mathrm{~g} \mathrm{~L}^{-1}$ \\
pH & 3,47 & DO $420 \mathrm{~nm}$ & 0,261 \\
Extrato seco & $20,40 \mathrm{~g} \mathrm{~L}^{-1}$ & DO 520nm & 0,320 \\
Açúcares redutores & $2,57 \mathrm{~g} \mathrm{~L}^{-1}$ & DO 620nm \\
Extrato seco reduzido & $18,83 \mathrm{~g} \mathrm{~L}^{-1}$ & Intensidade de cor \\
Álcool/Extrato seco reduzido & 4,81 & Matiz & 0,041 \\
Cinzas & $2,00 \mathrm{~g} \mathrm{~L}^{-1}$ & & 0,621 \\
\hline
\end{tabular}

${ }^{1}$ A análise de regressão polinomial não teve efeito significativo sobre as variáveia avaliadas com a probabilidade de $5 \%$ de erro. 
Tabela 2 - Regressão polinomial da análise sensorial - visão, olfato e gosto/sabor - do vinho Merlot jovem em função de crescentes produtividades do vinhedo. Bento Gonçalves, RS, 2000.

\begin{tabular}{|c|c|c|}
\hline Variável & Equação & Significância \\
\hline \multicolumn{3}{|l|}{ Visão } \\
\hline Limpidez (VLI) &.-- & NS \\
\hline Intensidade (VIN) & $Y=46+14,9 X-2,76 X^{2}$ & $* *$ \\
\hline Matiz (VMA) & $Y=44-2,6 X$ & $* *$ \\
\hline \multicolumn{3}{|l|}{ Olfato } \\
\hline Intensidade (OIN) & $Y=49+9,1 X-1,60 X^{2}$ & $*$ \\
\hline Fineza $(\mathrm{OFI})$ & $Y=49+2,6 X^{2}$ & $*$ \\
\hline Tipicidade (OTI) & $Y=48+2,3 X$ & $*$ \\
\hline Franqueza (OFA) & $Y=51+2,1 X$ & $*$ \\
\hline Pimentão verde (OPV) & $Y=8+15,8 X-3,12 X^{2}$ & $* *$ \\
\hline Frutado (OFR) &.-- & NS \\
\hline Especiaria (OEP) &.-- & NS \\
\hline Vegetal (OVE) & $Y=18+13,8 X-2,92 X^{2}$ & $*$ \\
\hline \multicolumn{3}{|l|}{ Gosto/Sabor } \\
\hline Intensidade (GIN) & $Y=44+12,6 X-2,18 X^{2}$ & $*$ \\
\hline Corpo (GCP) & $Y=48+2,2 X$ & $*$ \\
\hline Adstringência (GAS) &.-- & NS \\
\hline Acidez (GAC) &.-- & NS \\
\hline Amargor (GAM) &.-- & NS \\
\hline Salgado (GSA) &.-- & NS \\
\hline Equilíbrio (GEQ) & $Y=49+2,3 X$ & $*$ \\
\hline Qualidade (GQU) &.-- & NS \\
\hline Tipicidade (GTI) & $Y=48+2,1 X$ & $*$ \\
\hline Persistência (GPE) &.-- & NS \\
\hline Pimentão verde (GPV) &.-- & NS \\
\hline Frutado (GFR) & $Y=36+3,0 X$ & $*$ \\
\hline Especiaria (GEP) &.-- & NS \\
\hline Vegetal (GVE) &.-- & NS \\
\hline
\end{tabular}

$\mathrm{NS}=$ não significativo $; *$ = significativo a $5 \%$ de probabilidade $; *=$ significativo a $1 \%$ de probabilidade.

solar captada pelo dossel vegetativo, a variedade de uva, as práticas culturais executadas no vinhedo e no processo de elaboração do vinho, o terroir, a relação superfície foliar/peso fresco do fruto e a relação produção de uva/peso da poda (KLIEWER \& DOKOOZLIAN, 2005).

Verões quentes e chuvosos e solos em geral pouco profundos que caracterizam a Serra Gaúcha, favorecem a absorção praticamente constante e em volumes consideráveis de água do solo pelas raízes, a qual é transportada através do xilema até as folhas, o que induz vigor às videiras. Além disso, a água transportada ao fruto dilui o mosto. Esta diluição é tão importante que, a partir de uma determinada produtividade, a composição do mosto e do vinho não apresenta diferença significativa. Esta resposta da videira a fatores ambientais e a práticas enológicas evidencia um dos princípios enunciados por WINKLER et al. (1974), que afirmam que cada videira possui uma certa capacidade, ou seja, pode produzir uma determinada quantidade de uva com uma determinada qualidade para a finalidade desejada.

Sabe-se que, em geral, existe um estreito relacionamento entre a produtividade do vinhedo e a composição química do mosto da uva e do vinho (WINKLER, 1954; SINTON et al., 1978; BOULTON et al., 1998; SOMERS, 1998). Entretanto, esta relação nem sempre é evidente, pois há resultados que mostram que o aumento da concentração do açúcar na uva não é proporcional à diminuição da produtividade do vinhedo (FREEMAN, 1983; OUGH \& NAGAOKA, 1984; BRAVDO et al., 1985). Se esses resultados são válidos para a relação produtividade $x$ açúcar do mosto da uva, é lícito concluir que o vinho terá teores mais elevados de etanol. De fato, produtividades mais baixas favorecem vinhos com concentrações mais elevadas de seus principais constituintes, como é o caso do álcool, do extrato seco, das cinzas e dos polifenóis. Certos trabalhos, contudo, constatam que há um efeito muito pequeno, ou mesmo inexistente, sobre a qualidade do vinho (LOINGER \& SAFRAN, 1971; EAWART et al., 1985; JACKSON \& LOMBARD, 1993). Mais recentemente, pesquisas estudando vários fatores que exercem um papel essencial sobre a fisiologia da videira evidenciam que se pode aumentar sua produtividade e, inclusive, a qualidade da uva (BOULTON et al., 1998). Entretanto, tem-se que considerar a durabilidade das plantas, que seria diminuída pelas altas produtividades dos vinhedos. Para que isso não ocorra, são necessárias maciças fertilizações, o que implica aumento dos custos de produção da uva.

Esses resultados mostram que não se pode simplesmente adotar resultados de pesquisa de diferentes regiões vitivinícolas do mundo e aplicá-los no Brasil, como é o caso da Serra Gaúcha que possui características edáficas, climáticas e culturais específicas. É importante considerar, também, que podem existir terroirs onde a baixa produtividade do vinhedo favorece a qualidade da uva e do vinho. Para isso, faz-se necessário desenvolver pesquisas que harmonizem, para cada condição, a maximização da produtividade do vinhedo e a qualidade da uva e do vinho. Não se deve, entretanto, esquecer a rentabilidade da propriedade vitícola e a preferência do consumidor por produtos com características específicas. 


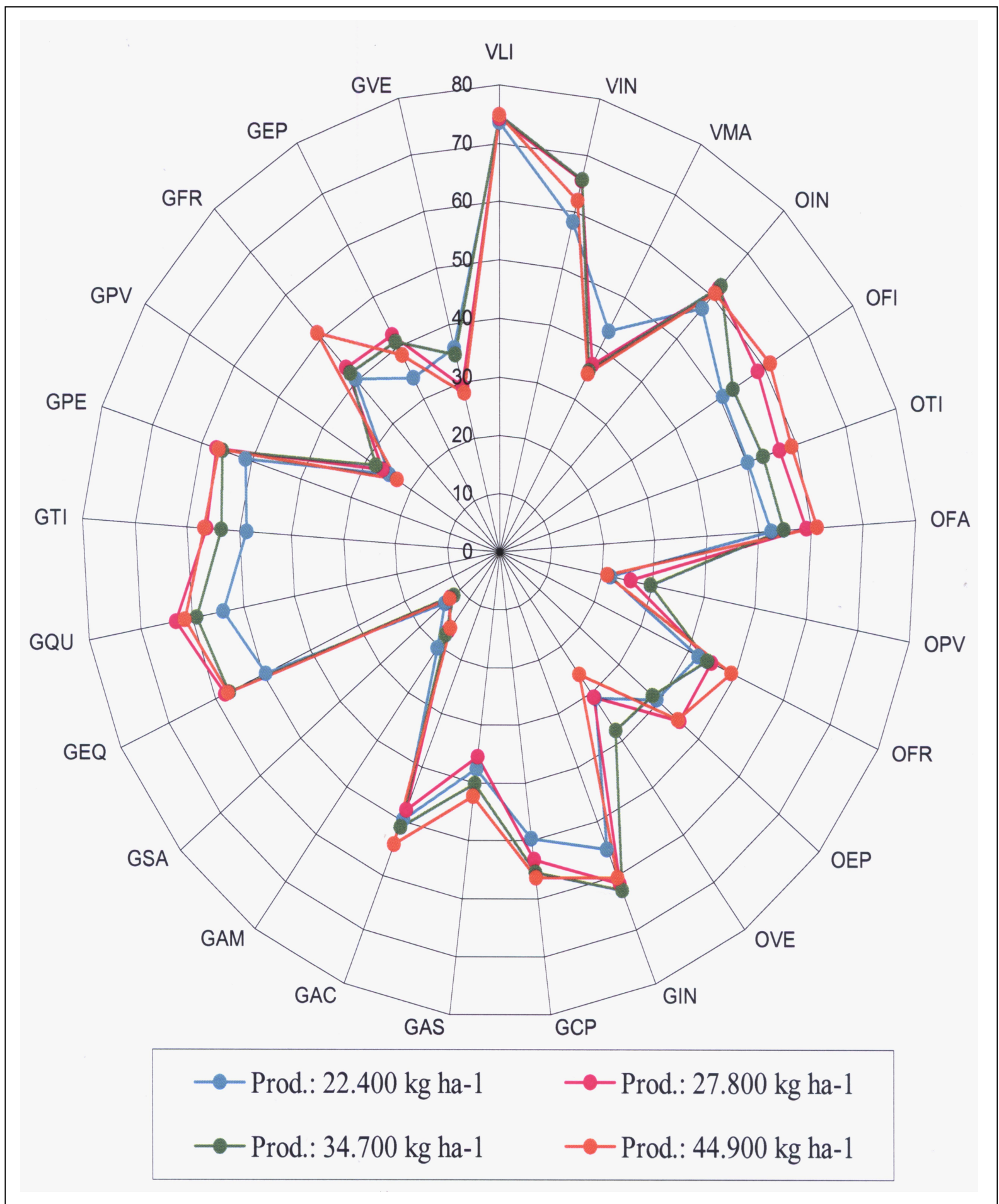

$\mathrm{V}=\underline{\text { Visão}} ; \mathrm{O}=\underline{\text { Olfato }} ; \mathrm{G}=\underline{\text { Gosto } / \text { Sabor }}$

$\mathrm{LI}=$ Limpidez; IN = Intensidade; MA = Matiz; FI = Fineza;

TI = Tipicidade $;$ FA = Franqueza $;$ PV = Pimentão verde;

$\mathrm{FR}=$ Frutado $; \mathrm{EP}=$ Especiaria $; \mathrm{VE}=$ Vegetal $; \mathrm{CP}=\mathrm{Corpo}$

$\mathrm{AS}=$ Adstringência; $\mathrm{AC}=$ Acidez; $\mathrm{AM}=$ Amargor; $\mathrm{SA}=$ Salgado;

$\mathrm{EQ}=$ Equilíbrio; $\mathrm{QU}=$ Qualidade $; \mathrm{PE}=$ Persistência . 


\section{CONCLUSÕES}

Em vinhedos de alta produtividade, seu aumento não teve efeito significativo sobre as variáveis físico-químicas do vinho Merlot jovem.

Quanto ao seu efeito sobre as características sensoriais, houve efeito significativo sobre as variáveis da visão, do olfato e do gosto/sabor. Os vinhos elaborados com uvas provenientes de vinhedos com a menor produtividade apresentaram-se visualmente com matiz vermelho-violáceo mais acentuado; mais francos, finos e típicos no olfato; e com gosto mais equilibrado, típico, frutado e mais encorpados. Os vinhos de média produtividade tiveram cor mais intensa; olfato também mais intenso, mas com notas vegetais e de pimentão verde mais acentuadas; gosto mais intenso. As demais variáveis sensoriais não foram afetadas pela produtividade do vinhedo.

\section{AGRADECIMENTOS}

Os autores agradecem a colaboração do engenheiro agrônomo Agliberto Bianchi e da enóloga Catiane Dall'Agnol.

\section{REFERÊNCIAS}

BOULTON, R.B. et al. Principles and practices of winemaking. Gaithersburg: Aspen, 1998. 604p.

BRAVDO, B. et al. Effect of crop level on growth, yield, and wine quality of a high yielding Carignane vineyard. American Journal of Enology and Viticulture, v.35, n.4, p.247-252, 1984.

BRAVDO, B. et al. The effect of crop level and crop load on growth, yield, must and wine composition, and wine quality of Cabernet Sauvignon. American Journal of Enology and Viticulture, v.36, n.2, p.125-131, 1985.

COLLALTO, G. di et al. Risultati di recherche sul diradamento dei grappoli della vite in ambiente collinare toscano. Vignevini, v.18, n.7/8, p.39-41, 1991.

EAWART, A.J.W. et al. The effects of light pruning, irrigation and improved management on wine quality of the Vitis vinifera cv. Riesling. Vitis, v.24, n 4, p 209-217, 1985.

FREEMAN, B.M. Effects of irrigation and prunning of Shiraz grapevines on subsequent red wine pigments. American Journal of Enology and Viticulture, v.34, n.1, p.23-26, 1983.

GRASSL, J. et al. Measurements of canopy formation and yield reduction with the grape cultivars 'Zweigelt' and 'Blauer Burgunder' ('Pinot Noir'). II. Effects on the contents of must and wine. Mitteilungen Klosterneuburg, Rebe und Wein, Obstbau und Fruchteverwertung, v.52, n.1/2, p.10-20, 2002.

JACKSON, D.I.; LOMBARD, P.B. Environmental and management practices affecting grape composition and wine quality - A review. American Journal of Enology and Viticulture, v.44, n.4, p.409-430, 1993.

KIEFER, W.; WEBER, M. Ausduennen zur qualitaetssteigerung. Deutsche Weinbau, n.14, p.24-28, 1994.

KLIEXR, W.M.; DOKOOZLIAN, N.K. Leaf area/crop weight ratios of grapevines: influence on fruit composition and wine quality. American Journal of Enology and Viticulture, v.56, n.2, p.170-181, 2005.

LAVIN, A.A. et al. Load levels in young 'Chardonnay' vineyards and their effects on yield and wine quality. Agricultura Tecnica, v.61, n.1, p.26-34, 2001.

LOINGER, C.; SAFRAN, B. Interdépendance entre le rendement, la maturation des raisins et la qualité des vins. Annales de Technologie Agricole, v.20, n.3, p.225-240, 1971

NAOR, A. et al. Shoot and cluster thinning influence vegetative growth, fruit yield, and wine quality of 'Sauvignon blanc' grapevines. Journal of the American Society for Horticultural Science, v.127, n.4, p.628-634, 2002.

OUGH, C.S.; NAGAOKA, R. Effect of cluster thinning and vineyard yields on grape and wine composition and wine quality of Cabernet Sauvignon. American Journal of Enology and Viticulture, v.35, n.1, p.30-34, 1984.

REYNOLDS, A.G. et al. Effect of shoot density and crop control on growth, yield, fruit composition, and wine quality of Seyval blanc grapes. Journal of the American Society for Horticultural Science, v.111, n.1, p.56-63, 1986.

RIBÉREAU-GAYON, J. et al. Sciences et techniques du vin. Paris: Dunod, 1976. V.1.

RIBÉREAU-GAYON, P.; STONESTREET, E. Dosage de tanins $\mathrm{du}$ vin rouge et détermination de leur structure. Chimie Analytique, v.48, n.4, p.188-196, 1966.

RIBÉREAU-GAYON, P.; STONESTREET, E. Le dosage des anthocyanes dans les vins rouges. Bulletin de la Société Chimique de France, v.9, n.419, p.2.649-2.652, 1965.

SCHALKWYK, D. van et al. Effect of bunch removal on grape composition and wine quality of Vitis vinifera $\mathrm{L}$. cv. Chardonay. South African Journal for Enology and Viticulture, v.16, n.2, p.15-25, 1995.

SINTON, T.H. et al. Grape juice indicators for prediction of potential wine quality. I. Relationship between crop level, juice and wine composition and wine sensory rating and scores. American Journal of Enology and Viticulture, v.29, n.4, p.267-271, 1978.

SOMERS, C. The wine spectrum: an approach towards objective definition of wine quality. Adelaide: Winetitles, 1998. $135 \mathrm{p}$.

WINKLER, A.J. Effects of overcropping. American Journal of Enology and Viticulture, v.5, n.1, p.4-12, 1954.

WINKLER, A.J. et al. General viticulture. Berkeley: University of California, 1974. 710p. 